\title{
The Role of the Filler Surface Chemistry on the Dielectric and Thermal Properties of Polypropylene Aluminium Nitride Nanocomposites
}

\author{
Xinyu Wang, Thomas Andritsch, George Chen and Suvi Virtanen \\ Tony Davies High Voltage Laboratory \\ University of Southampton \\ Southampton, UK
}

\begin{abstract}
In nanocomposites, different surface states of nanoparticles can potentially provide different interactions with the base polymer and in turn change the bulk properties. Aluminium nitride (AIN) nanoparticles were surface functionalised with three different silane coupling agents (SCAs) with varying organofunctional and hydrolysable groups. The effects of the filler surface chemistry on the resulting AIN/polypropylene (PP) nanocomposites were examined and compared with an unfilled reference system. It is observed that different organofunctional groups can provide different nucleating effects and the dispersion states of nanoparticles while the hydrolysable group is not the dominant factor. The dielectric spectroscopy results show the hydrolysable group of SCA will also result in a difference of the interphase since the trimethoxy silane treated systems show much higher imaginary permittivity than the triethoxy silane treated systems when the frequency is below $1 \mathrm{~Hz}$. The grafted organofunctional layer on the particle surface can provide a significant improvement of the thermal conductivity of the composite materials, e.g. $15 \%$ improvement in thermal conductivity was observed when adding $10 \mathrm{wt} \%$ methacrylate silanes treated nano-AIN into PP, while the untreated counterpart only has $5 \%$ improvement.
\end{abstract}

Index Terms - nanocomposites, interphase, polypropylene, aluminium nitride, silane coupling agent, dielectric spectroscopy, thermal conductivity

\section{INTRODUCTION}

NANODIELECTRICS are the polymer nanocomposites used for electrical insulation within which nano-sized fillers are added to a polymer or polymer blend, traditionally at only a few weight per cent $(w t \%)$. The concept of "nanodielectrics" [1] was first introduced as "nanometric dielectrics" by Lewis in 1994 [2]. Nevertheless, the research on nanodielectrics did not receive much attention until promising results were shown, such as suppressed space charge accumulation, as presented by the pioneer experimental work of Nelson and Fothergill et al. in 2002 [3]. However, while the potential benefits of nanodielectrics have been exploited over the last twenty years, there are plenty of inconsistent or contradictory results that impede the thorough understanding on how nanoparticle interact with the polymer.

Indeed, when discussing the main factor that can dominate the property changes caused by adding nanoparticles into the polymer dielectrics, the notion of the large interfacial region

Manuscript received on 20 August 2018, in final form 24 January 2019, accepted xx Month 20yy. Corresponding author: X. Wang. between nanoparticles and the base polymer, giving rise to the interphase volume, plays a significant role and is thus highlighted by many researchers $[2,4,5]$. Therefore, the dispersion of nanoparticles and how to reduce the agglomerations to maximise surface area are considered to be essential issues for nanodielectrics. This leads to a broad application of surface functionalisation in nanodielectrics research [6-8]. It is considered that the surface functionalisation can improve the nanoparticles dispersion by reducing the surface energy or by forming chemical bonding with the matrix material.

The silane coupling agent (SCA) is mainly used for surface functionalisation of inorganic particles, and the surface chemistry of nanoparticles can be altered dramatically after grafting such molecules onto the surface $[9,10]$. The typical structure of SCA is shown in Figure 1a. The hydrolysable groups will chemically bond on the surface of the nanoparticles and are generally considered to have less impact on the interphase properties. Meanwhile, the organofunctional group has received more attention by researchers in their selection of SCA [9, 10]. Epoxide silanes are mainly used in epoxy nanocomposites, as they can form covalent bonds with the matrix [11]. However, polymers like polyethene (PE) and 


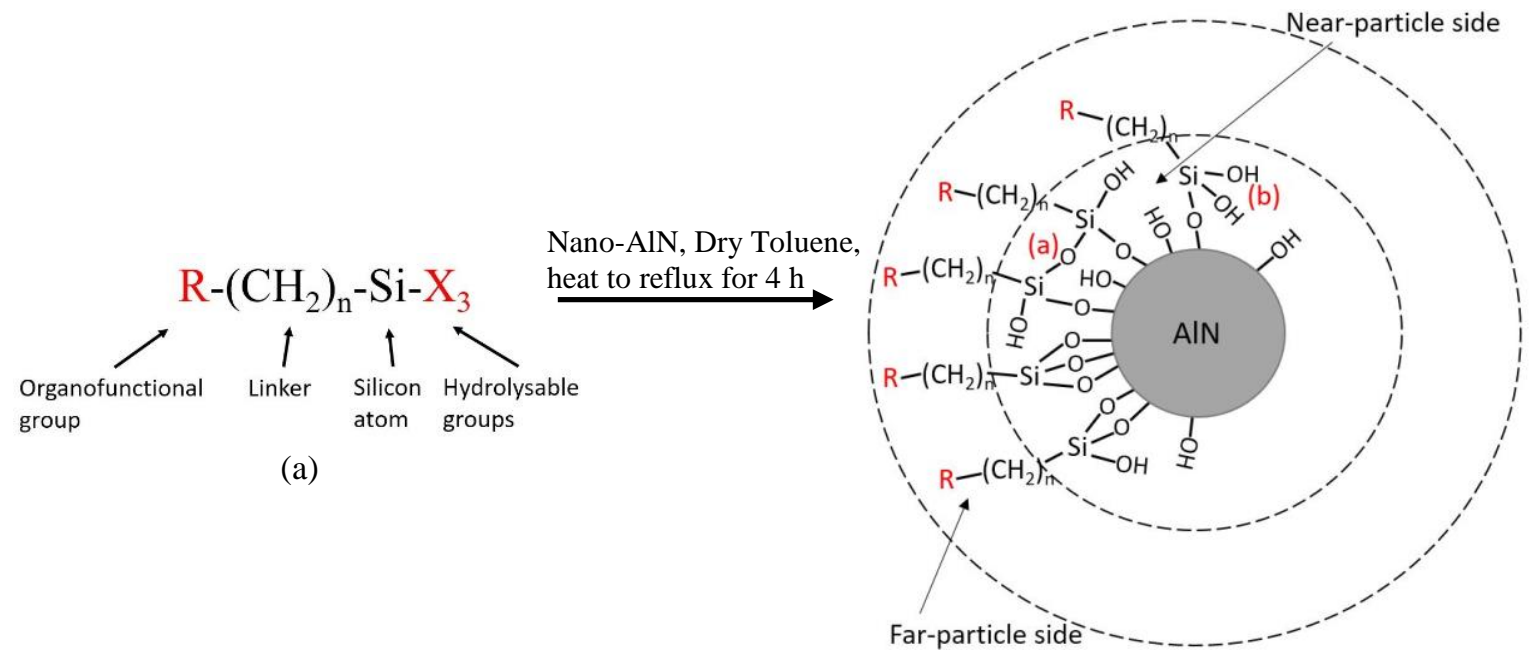

(b)

Figure 1. (a) General chemical formula of SCA and (b) the proposed surface structure of the functionalised nanoparticle.

polypropylene (PP) with a carbon chain structure, which is nonpolar, functional group free and low unsaturation, are unlikely to interact chemically with any SCA. It is generally believed that alkyl silanes, such as propyl (C3) and octyl (C8) silanes can thermodynamically increase the compatibility between the nanoparticles and carbon chains due to their similar carbon chain structures [6, 12]. Although some studies believe methacrylate silanes can form covalent bonds with PP [10, 13], there is no convincing evidence to show that any SCA can durably bond to PP.

It has been reported that different surface functionalisations can affect bulk properties [14, 15]. On the far-particle side (organofunctional group, as shown in Figure 1b), Roy et al point out that nanocomposites with non-polar SCAs surface modified particles will have lower activation energy derived from dielectric spectroscopy than the sample that is modified by a polar SCA [15]. However, some studies claimed that the bulk properties have less dependence on the minor variation of the organofunctional side, such as the chain length [12]. The above work also provides some evidence that there is an influence of SCA on the near-particle side (as shown in Figure 1b). For example, the consumption of the hydroxyl group by SCA can reduce water absorption, which can significantly affect the electrical properties of nanocomposites.

The hydrolysable groups are usually alkoxy group with one or two carbons in length, namely methoxy $\left(-\mathrm{CH}_{3} \mathrm{O}\right)$ and ethoxy $\left(-\mathrm{C}_{2} \mathrm{H}_{5} \mathrm{O}\right)$. Not much research has been done on the effect of the hydrolysable groups. It might be because the hydrolysable groups will mainly be removed during the surface functionalisation process, and the final silane functionalised nanoparticles may only have few hydrolysable groups remained on the surface. However, the hydrolysable groups will affect the hydrolysis rate and grafting mechanism, which may have a great impact on the surface chemistry of nanoparticles. Thus, SCAs with different hydrolysable groups are included in this work. Indeed, it can hopefully act as a probe that can reveal the role of the surface chemistry (near-particle side) of SCA treated particles on the composite materials.

The SCA grafting mechanisms on the near-particle side are classified as hydrous and anhydrous mechanisms [11]. Some work has demonstrated that a thicker layer of silane, formed by condensation on the nano-silica surface when applying the hydrous method, can result in larger agglomerations and higher relative permittivity [11]. This work highlighted the chemistry and structural complexity of the nanoparticle surface. The anhydrous method was selected in this work, as it can provide better-controlled specimens, as the condensation can be restricted.

Regarding the application of PP-based materials, it is considered to be a great potential for recyclable HVDC cable insulations [7, 16]. However, the poor thermal conductivity of PP impedes the PP cable to reach higher operating temperature [16]. Many studies show that high thermal conductive nanoparticles, such as aluminium nitride (AlN) and boron nitride $(\mathrm{BN})$, can improve the thermal conductivity of the matrix material $[7,8,17]$.

Previous work [18] demonstrated the effect of the surface chemistry of AIN nanoparticles on the AC breakdown strength, in this work, nano-AIN was treated with three different SCAs, with variation in both organofunctional and hydrolysable groups, which were then solvent blended with PP. The aim is to have a better insight into the effect of the filler surface chemistry on the dielectric response and thermal properties of $\mathrm{PP} / \mathrm{AlN}$ nanocomposites, from both the far- and near-particle side aspects.

\section{EXPERIMENTAL}

\subsection{MATERIALS AND SAMPLE PREPARATION}

Full details of the materials used as well as surface functionalisation and sample preparation methods are published in previous work [18]. The important details are listed below. Nano-AlN nanoparticles $(50 \mathrm{~nm})$ were obtained from Aladdin Inc. Before being blended with isotactic polypropylene (iPP), 427861 Aldrich, they were surface functionalised by three different SCAs: 3-(trimethoxysilyl)-propyl methacrylate (MPTS-M), the trimethoxy(octyl)silane (C8-M) and the triethoxy(octyl)silane (C8-E). The differences between above SCAs are listed in Table 1. 
Table 1. The differences between SCAs applied in terms of their functional group on both sides.

\begin{tabular}{c|c|c}
\hline SCA & $\begin{array}{c}\text { Organofunctional } \\
\text { group }\end{array}$ & $\begin{array}{c}\text { Hydrolysable } \\
\text { group }\end{array}$ \\
\hline MPTS-M & Methacrylate & Methoxy \\
\hline C8-M & Octyl & Methoxy \\
\hline C8-E & Octyl & Ethoxy \\
\hline
\end{tabular}

The loading ratios of 5 and $10 \mathrm{wt} \%$ were chosen since a low content of fillers was reported to have an insignificant effect on the thermal conductivity of the composite material. A total of $10 \mathrm{~g}$ of nanocomposites were prepared for each system. The nanocomposites systems were denoted as "SCA-loading ratio". For example, the nanocomposite that contains $5 \mathrm{wt} \%$ of $\mathrm{C} 8-\mathrm{E}$ treated nano-AlN nanoparticles was denoted as "C8-E-5". Unfilled iPP and iPP filled with 5 and $10 \mathrm{wt} \%$ of untreated nano-AlN were denoted as "PP", "PP-5" and "PP-10", respectively.

Here, 9.5 or $9 \mathrm{~g}$ of iPP was dissolved in $100 \mathrm{ml}$ of boiling xylene. For untreated nano-AIN, either 0.5 or $1 \mathrm{~g}$ of powder was dispersed in $20 \mathrm{ml}$ of toluene followed with the help of a probe sonicator. The surface functionalised nano-AlN were ready to use after the surface functionalisation procedure described in [18]. After iPP pellets were well dissolved, the iPP/xylene was removed from heat and allowed it to become slightly gel before the nano-powder/toluene mixture was added. The resulting mixture was vigorously stirred until it becomes rubber-like. It was then placed in a fume cupboard to evaporate the majority of solvent for 24 hours. Before the master blend was pressed into samples for each test, it was pressed by a hydraulic press to remove the bubbles and cavities inside and dried in a vacuum oven at $80^{\circ} \mathrm{C}$ for 3 days to remove the solvent residues.

\subsection{CHARACTERISATION}

Fourier-transform infrared spectroscopy (FTIR) analysis was performed to examine the surface chemistry of nano-AlN, $2 \mathrm{mg}$ of powder was examined by a Nicolet iS5 FTIR Spectrometer with a wavelength ranging from 500 to $4000 \mathrm{~cm}^{-1}$. The tests were running in ATR mode and the ambient air background was measured for calibration before each test.
Thermogravimetric analysis (TGA) data was obtained by heating $5 \mathrm{mg}$ of samples from 100 to $600{ }^{\circ} \mathrm{C}$ at a heating rate of $20{ }^{\circ} \mathrm{C} / \mathrm{min}$ to verify the real loading ratio and from 100 to $900{ }^{\circ} \mathrm{C}$ at a heating rate of $10^{\circ} \mathrm{C} / \mathrm{min}$ to examine the untreated and treated nano-AlN powders. Scanning electron microscopy (SEM) was performed on the film samples treated by an etching procedure described in previous work [19]. The samples were placed on an aluminium slab and gold coated by an Emitech K550X coater. Differential scanning calorimetry (DSC) analysis was done by a Perkin Elmer DSC-7. The temperature scan method was set up for all tested polymeric samples as follows: the sample was heated from 20 to $200{ }^{\circ} \mathrm{C}$ at $10{ }^{\circ} \mathrm{C} / \mathrm{min}$ and kept at $200{ }^{\circ} \mathrm{C}$ for $5 \mathrm{~min}$ to remove the thermal history. Then the sample was cooled to $20{ }^{\circ} \mathrm{C}$ to obtain the crystallisation curve. The temperature was again raised to $200{ }^{\circ} \mathrm{C}$ at $10^{\circ} \mathrm{C} / \mathrm{min}$ to get the heating curve.

Dielectric spectroscopy was performed for polymeric samples of $0.21 \mathrm{~mm}$ thickness. Samples with gold-coated surfaces were tested by a parallel test cell with $32 \mathrm{~mm}$ diameter electrodes at $90{ }^{\circ} \mathrm{C}$. The complex permittivity was measured from 0.01 or 0.1 to $10^{5} \mathrm{~Hz}$ with a Solartron 1296 interface linked to a Schlumberger SI 1260 impedance-gain-phase analyser.

The thermal conductivity of each system was performed by a steady-state technique based on the guarded hot plate method [20]. A thin film flux sensor from OMEGA Engineering, HFS4 , was used. Flat samples with $3 \mathrm{~mm}$ in thickness and $5 \mathrm{~cm}$ in diameter were tested 3 times each. Another neat PP sample with a thickness of $2.66 \mathrm{~mm}$ was used for calibration.

\section{RESULTS}

\subsection{FTIR ANALYSIS}

The FTIR results of MPTS-M, C8-M and C8-E treated nanoAlN are shown in Figure 2. These traces have been vertically offset for clarity of presentation. The bands of MPTS-M curve at 1718 and $1637 \mathrm{~cm}^{-1}$ are associated with the stretching vibration of $\mathrm{C}=\mathrm{O}$ and $\mathrm{C}=\mathrm{C}$ groups $[14,21]$ and the two bands at 1297 and $1170 \mathrm{~cm}^{-1}$ are assigned to $-\mathrm{C}-\mathrm{O}-\mathrm{C}$ group [21]. From



Figure 2. FTIR spectra of MPTS-M, C8-M and C8-E treated nano-AIN. 
the spectra of C8-M and C8-E, three comparable peaks are found at 2959, 2922 and $2853 \mathrm{~cm}^{-1}$, which correspond to the stretching vibration of $\mathrm{C}-\mathrm{H},-\mathrm{CH}_{3}$ and $-\mathrm{CH}_{2}$. All spectra have a distinct wide peak from 968 to $1142 \mathrm{~cm}^{-1}$, related to the presence of Si-O-Si, Si-O-C and Si-OH. The peak at $1458 \mathrm{~cm}^{-1}$ is related to the bending vibration of $-\mathrm{CH}_{3}[14,21]$. Combined with the TGA results in [18], there is little doubt that the SCA bonded on the surface of nano-AIN. The change of the surface chemistry by different organofunctional groups can be clearly distinguished.

However, the hydrolysable group of SCA shows no effect on the FTIR spectra. The hydrolysable group is expected to provide different grafting mechanisms of SCA onto the particle surface. The feature of the near-particle side, $\mathrm{Si}-\mathrm{O}-\mathrm{Si}, \mathrm{Si}-\mathrm{O}-\mathrm{C}$, and $\mathrm{Si}-\mathrm{OH}$ falls into a wide peak, which is difficult to differentiate. That means, by far, only the far-particle side was characterised by FTIR analyses.

\subsection{COMPOSITION AND MORPHOLOGY}

The TGA curves of as-received and functionalised nano-AlN were presented in related work [18], which showed that SCAs were effectively bonded onto the surface of nanoparticles. The TGA curves for the different PP/AlN nanocomposites are shown in Figure 3. All $5 \mathrm{wt} \%$ samples have an actual loading ratio of $4.6 \pm 0.5$ and $9.8 \pm 0.8 \mathrm{wt} \%$ for $10 \mathrm{wt} \%$, which are as expected. The thermal stability of PP is dramatically improved by blending nano-AlN which increasing the onset of weight loss by more than $20^{\circ} \mathrm{C}$. This is in agreement with $[7,12,19]$ that the nanoparticles can improve the thermal stability of the system. However, the thermal stability is independent of the surface chemistry of nanoparticles, which cannot be taken as the evidence of the increased interaction between surface organofunctional groups and the matrix. In the work of Hosier et al. [19], different fillers also result in comparable thermal stabilities, also with different surface chemistry states.

The SEM result of the unfilled PP is shown in Figure 4. A clear spherulitic crystalline structure can be found, and the size of the spherulitic crystalline structure ranges from 25 to $30 \mu \mathrm{m}$.

The SEM results of $5 \mathrm{wt} \%$ were presented in the related paper [18], and similar trends can be seen in $10 \mathrm{wt} \%$ composites. For

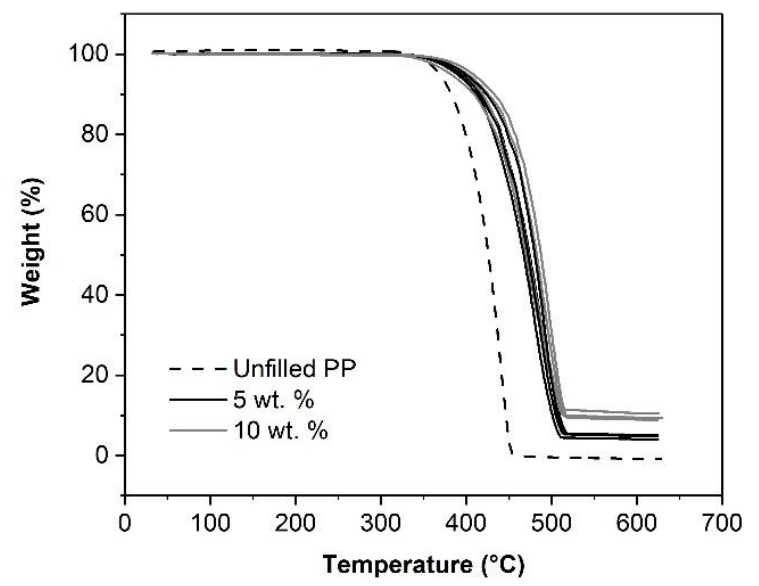

Figure 3. TGA curves for the different PP/AIN nanocomposites.
PP-10, large agglomerations around $10 \mu \mathrm{m}$ can be seen from Figure 5a, throughout the sample. Spherulitic crystalline structures of reduced size can be observed. This can be attributed to the nucleating effect of nanoparticles discussed below [19].

When comparing the SEM results of untreated and MPTS-M functionalised nano-AIN/PP systems from Figures 5a and 5b, the grafted MPTS-M silanes on nanoparticle surface have little effect on the dispersion. According to the SEM image of MPTS-M, C8-M and C8-E functionalised nano-AIN/PP systems in Figures $5 \mathrm{~b}-\mathrm{d}$, it can be observed that the silane functionalisation can somewhat improve the dispersion of the nano-AlN in PP and different SCAs will cause noticeable changes. When comparing MPTS-M and C8-M functionalised nano-AlN/PP systems, it seems that $\mathrm{C} 8$ groups have better performance in regard of improving the nano-AlN dispersion, because fewer 5-10 $\mu \mathrm{m}$ agglomerations and more 1-2 $\mu \mathrm{m}$ agglomerations can be found in $\mathrm{C} 8 \mathrm{-M}$ functionalised nanoAlN/PP system. Similar results can be found in C8-E functionalised nano-AlN/PP system, which indicates that different hydrolysable groups have little effect on the nanoparticles dispersion. In addition, no obvious spherulitic

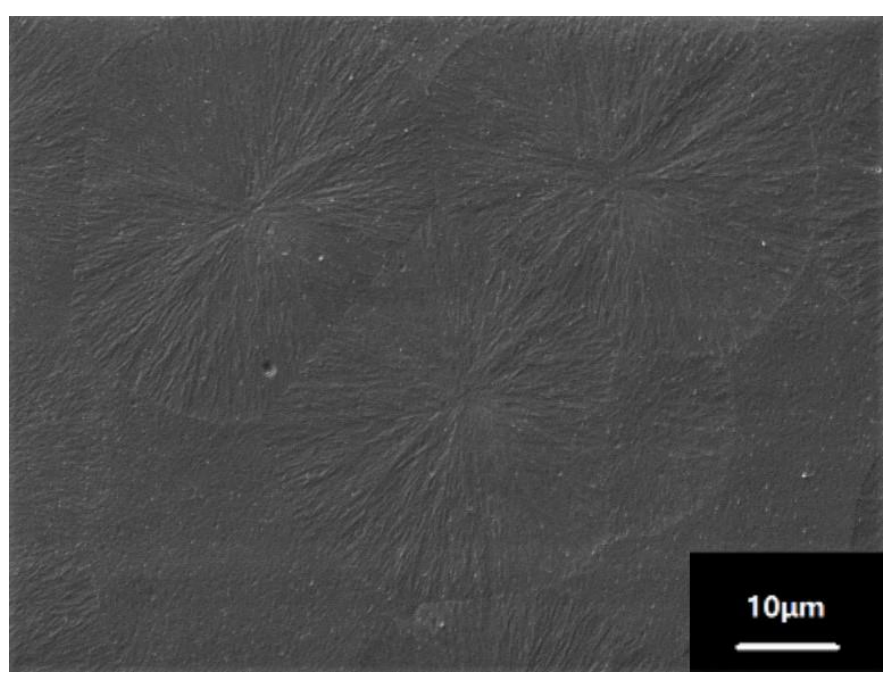

Figure 4. SEM image of base PP.



Figure 5. SEM images of (a) PP-10, (b) MPTS-M-10, (c) C8-M-10, (d) C8 E-10. 
(a)

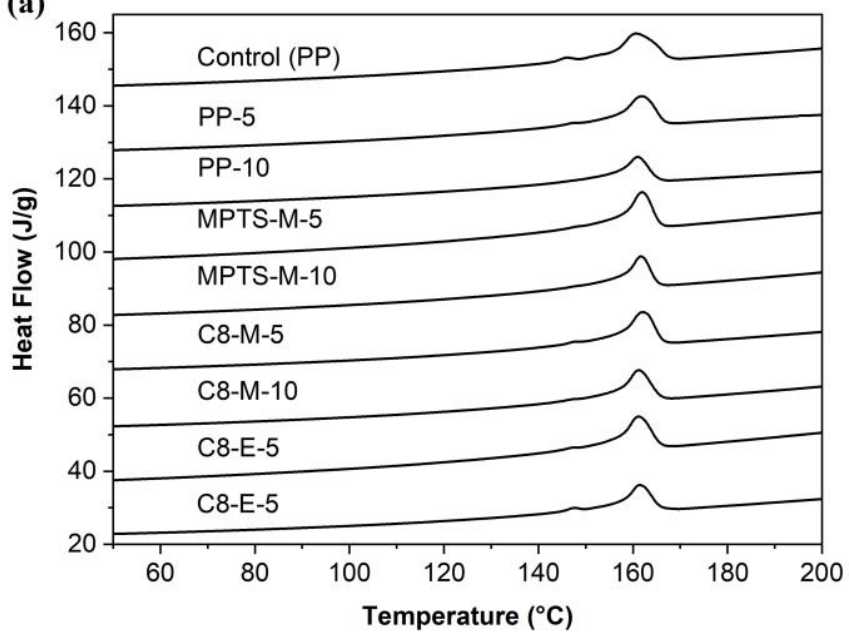

(b)

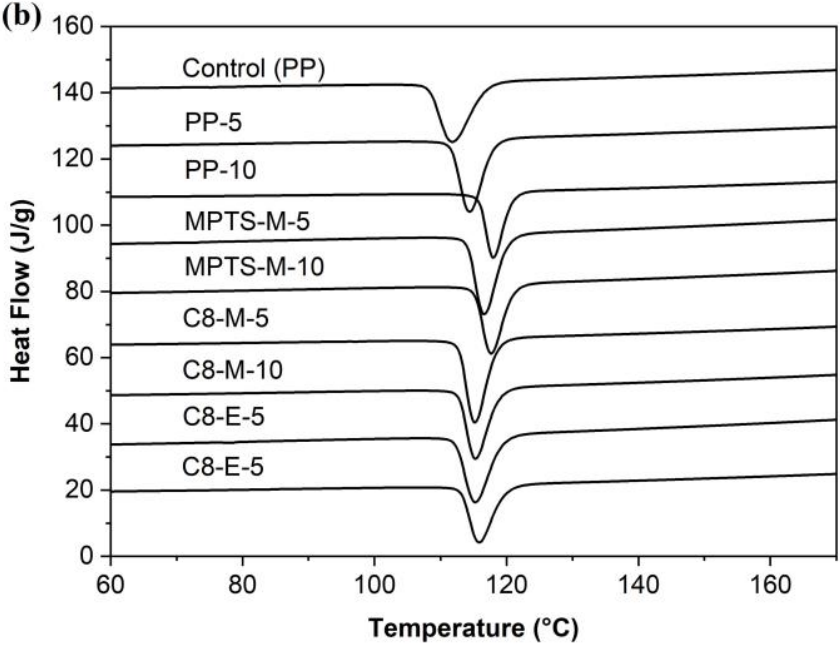

Figure 6. DSC heating and crystallisation curve of each dielectric system.

crystalline structures can be found in nanocomposites containing silane functionalised nano-AIN.

Figure 6 shows the DSC heating and crystallisation curve of each dielectric system. From heating curves, the melting point of each system seems unaffected by the presence of nano-AlN, although some nanocomposites show a small increase up to $2{ }^{\circ} \mathrm{C}$. From crystallisation traces, it can be seen that the untreated systems have higher crystallisation temperature than the reference iPP. The same has been observed before for nanocomposites with functionalised filler, and the reason for it was stated that nanoparticles can act as the nucleating agent and elevate the crystallisation temperature [19]. The crystallisation temperature of MPTS and C8 treated systems are less dependent on the loading ratio but can also present this nucleating effect. However, the C8-M and C8-E treated nanoparticles show weaker abilities to increase the crystallisation temperature than both MPTS treated and untreated nanoparticles. These results are consistent with works that use non-polar SCAs $[6,12]$. Since the untreated nanoparticles can be considered to have polar groups on the surface, like $-\mathrm{OH}$ and $-\mathrm{NH}_{2}$, it can be concluded that the polar surface of nanoparticles can provide a stronger nucleating effect. All nanocomposites have a decreased melting enthalpy and present dependency on the loading ratio rather than on the surface chemistry. This observation has been explained by the hindrance effect of nanoparticles on the growth of the crystalline structure $[6,19]$.

\subsection{DIELECTRIC SPECTROSCOPY}

Both the real and imaginary permittivity of the AlN/PP nanocomposites and unfilled PP measured at $90{ }^{\circ} \mathrm{C}$ are plotted in Figure 7. It can be observed that the real permittivity of filled systems is higher than the base PP, and further increases with loading concentration. When taking into consideration the uncertainty, \pm 0.1 , based on repeated measurements, all nanofilled systems have a comparable level of real permittivity. However, the upward turns for some samples at low frequencies appear.

The Havriliak Negami (HN) fitting [22] was applied to analyse the relaxation features on the dielectric spectra. The best fittings indicate that MPTS-M-5 and MPTS-M-10 have relaxation peak at $0.21 \mathrm{~Hz}$ and $0.05 \mathrm{~Hz}$, respectively. The best fittings for other systems cannot be reached after 400 times of integration based on the Levenberg-Marquardt algorithm, or hit the lower limit of the frequency $(0.01 \mathrm{~Hz})$. However, there is a distinct similarity between the response of MPTS-M-10 and C8-M-10. To further study the similarities observed in these two composites in Figure 7, the dielectric response of MPTS-M-10 and C8-M-10 were measured until $0.01 \mathrm{~Hz}$. Figure 8 shows the low-frequency features of the real and imaginary permittivity of C8-M-10 and MPTS-M-10. The data confirms the fitting results, where relaxation peak can be observed at about $0.05 \mathrm{~Hz}$ for MPTS-M10 while the imaginary part of C8-M-10 shows no peak, but rather a sharp upturn all the way up to $0.01 \mathrm{~Hz}$.

The increased imaginary permittivity at low frequencies could imply the percolation of the interphase, which results in the electrical conduction. The log-log plot of the real and imaginary permittivity of C8-M-10 shows the slope of the imaginary part from 0.1 to $0.01 \mathrm{~Hz}$ is -0.9 . This indicates the upturn of the imaginary permittivity at low frequencies of this system is likely to be the quasi-DC conductivity contribution, which is often related to overlapping water shells in nanodielectrics [23]. In MPTS-M functionalised nano-AlN/PP systems, the relaxation peak shifts to low frequency from $5 \mathrm{wt} \%$ to $10 \mathrm{wt} \%$. Similar behaviour with increased filler content has been reported previously [24]. The reason for this behaviour boils down to the abundance of the filler agglomerates present. The moving of dipoles such as water or polar groups from SCA inside them can be restricted [24].

For systems with untreated fillers, the increased real permittivity, as well as imaginary permittivity at low-frequency range, may indicate that the Maxwell-Wagner-Sillars interfacial polarization occurred. These can be related to the poor compatibility between nanoparticles and the PP matrix. The non-polar carbon chain and low unsaturation structure of PP will have low compatibility with polar groups like $-\mathrm{OH}$, which is also consistent with SEM images shown in Figure 5, where the untreated system has noticeable larger agglomerations compared to other systems. The hydroxyl groups on the particle surface can also absorb ambient humidity and a subsequent 

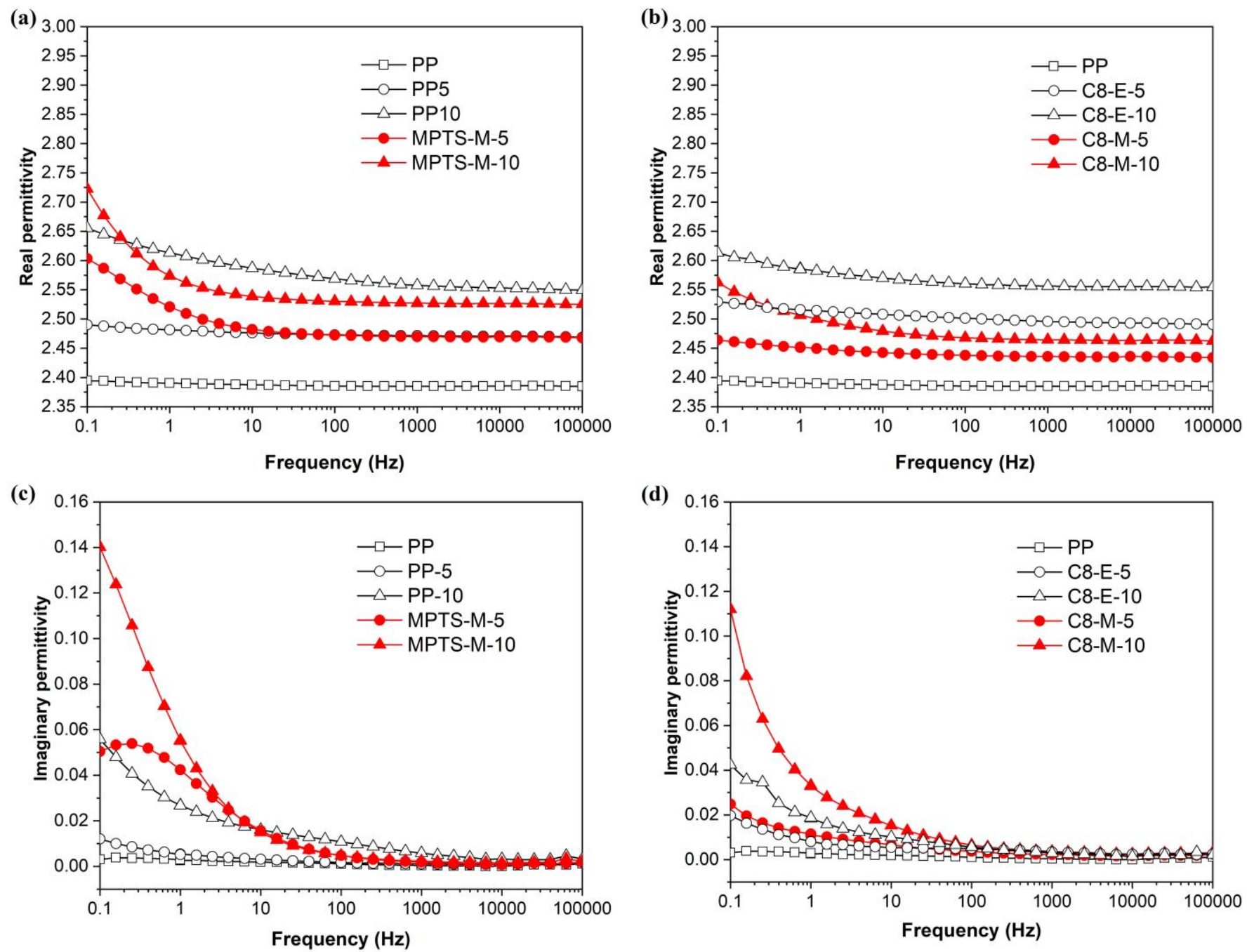

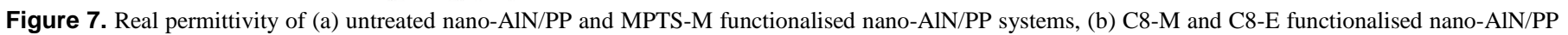

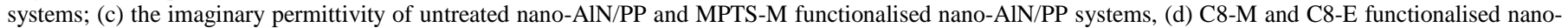
AlN/PP systems at $90^{\circ} \mathrm{C}$.

increase of the dielectric loss in the low-frequency range can be observed [6, 12, 19]. C8-E-10 shows the lower imaginary permittivity and flatter up-turn of real permittivity at low

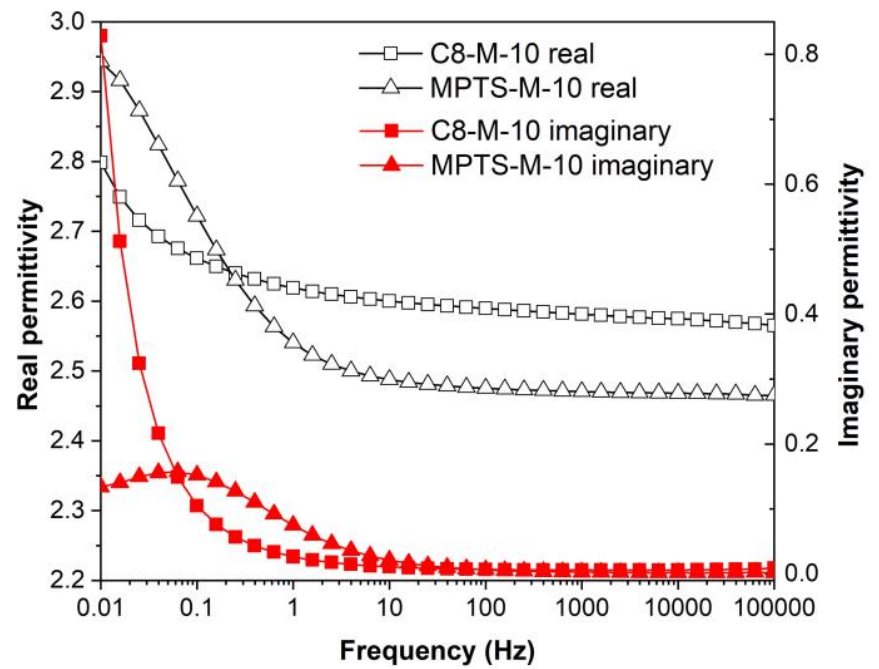

Figure 8. Real and imaginary permittivity of C8-M-10 and MPTS-M-10 at $90{ }^{\circ} \mathrm{C}$ (measured until $0.01 \mathrm{~Hz}$ ). frequencies than the untreated counterpart, PP-10. After functionalised by $\mathrm{C} 8$ silane, some hydroxyl groups are displaced by octyl groups which are non-polar and can improve the compatibility between the filler and matrix and reduce the Maxwell-Wagner-Sillars interfacial polarization. Also, the reduction of hydroxyl groups can lead to less water absorption effect and reduce the system loss [6, 19].

However, the C8-M treated systems are observed to have an even higher loss than the untreated systems and a distinct upturn of real permittivity at low frequencies (obvious difference can be found from the results of C8-M-10 and C8-E-10). Indeed, these features could be the superposition of the dipole relaxation, Maxwell-Wagner-Sillars interfacial polarization and quasi-DC conductivity. Compared to the C8-E treated systems, C8-M treated systems have comparable far-particle chemical structures. These results indicate that the near-particle side will also have a great impact on the bulk properties. The proposed near-particle surface structure of the functionalised nanoparticle shown in Figure $1 \mathrm{~b}$ can explain the observed difference between $\mathrm{C} 8 \mathrm{-M}$ and $\mathrm{C} 8 \mathrm{-E}$ treated systems. The different near-particle surface structure can originate from the following: (1) the condensation of SCAs (illustrated in Figure 
$1 \mathrm{~b}$, position (a)), which can result in an ill-defined surface structure [11], since the hydrolysable group of SCA can result in different condensation rates; (2) the un-bonded hydrolysable group will subsequently be hydrolysed by ambient moisture and form Si-OH on the near-particle side (illustrated in Figure 1b, position (b)). This means the SCA treated nanoparticles may have more hydroxyl groups compared to the untreated particles. Those hydroxyl groups on near particle side can result in the increased imaginary permittivity.

When linking the bulk properties to the surface chemistry as well as the interphase properties, the chemical structure of the particle surface should be well defined. Unfortunately, the commonly used techniques such as Raman spectroscopy and FTIR can only identify the far-particle side rather than the nearparticle side. Therefore, further study to formulate and characterise the interphase region between nanoparticles and matrix in both near and far particle sides are needed. There is a good example in [4] that shows that the X-ray photoelectron spectroscopy (XPS) could be used to identify the structure of near-particle side.

\subsection{THERMAL CONDUCTIVITY}

The thermal conductivity of each sample is shown in Figure 9. It is clear that: (1) the nano-AlN filled PP show higher thermal conductivity than the unfilled PP and all nanocomposites have monotonically increased thermal conductivity as a function of the loading ratio; (2) in functionalised systems, an increased thermal conductivity compared to untreated systems can be observed. Different surface treatments seem to result in a similar ability of the bulk to conduct the heat flow; (3) it is noticeable that PP filled with $10 \mathrm{wt} \%$ MPTS-M treated nano-AlN can have $14.8 \%$ of improvement over the unfilled PP, which is consistent with the work that blended $5 \mathrm{wt} \%$ of nano-AlN with PP [7] and $10 \mathrm{wt} \%$ of nano-AlN with epoxy [17], where 13.3 and $17.9 \%$ of thermal conductivity enhancement were achieved.

When incorporating the non-metallic material into a polymer, the interphase barrier between nanoparticles and polymer matrix is the main contribution to the phonon scattering [8]. The surface treatment can improve the compatibility between the nanoparticles and matrix material $[8,17]$. The surface treated

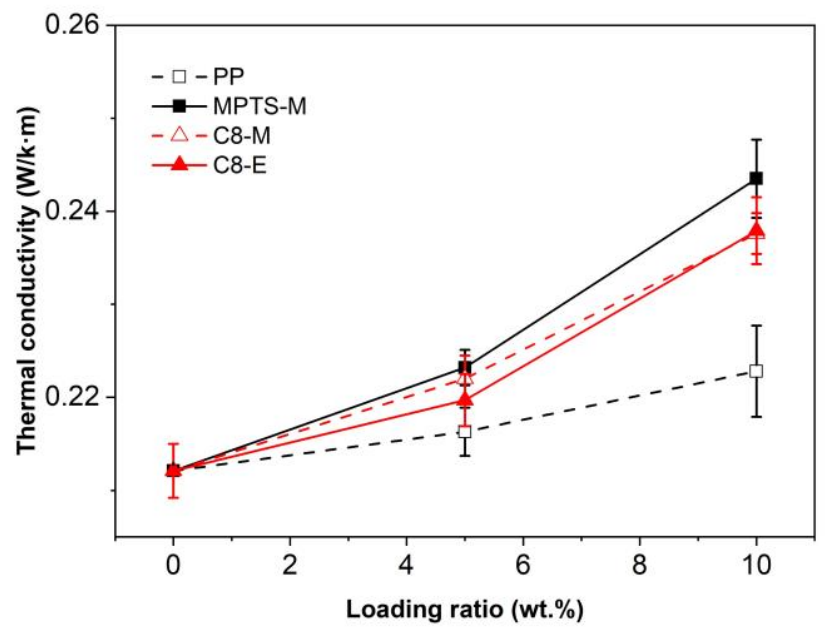

Figure 9. Thermal conductivity of each system. systems can be observed to have a significant improvement compared to the untreated systems. Without surfactant acting as a compatibiliser, the phase separation between nanoparticles and polymers can result in a strong phonon scattering phenomenon during the heat flow transmission. It is consistent with the SEM results in Figure 5 where the surface functionalisation can improve the compatibility between the nanoparticles and PP and an improved dispersion can be observed.

One interpretation of these results is that the organic layers on the particle surface weakens the scattering phenomenon and decreases the heat resistance of the interphase [25]. The thermal conductivity results here also show less dependence on the dispersion of nanoparticles. Thus, the results show the significant role the surface chemistry plays regarding the thermal conductivity of nanocomposites.

\section{CONCLUSIONS}

Eight nanocomposites with four different types of particle surface chemistry states and two loading ratios have been studied, along with unfilled samples as a control group. The surface functionalisation of nanoparticles shows a great effect on the morphology, thermal and dielectric properties of nanocomposites. The following conclusions can be drawn from the present work: The structure characterisation obtained by SEM shows that the spherulitic crystalline structures are disturbed by the introduction of nanoparticles. No distinguishable spherulitic crystalline structure can be observed in nanocomposites. Obvious aggregation can be seen in all samples, but $\mathrm{C} 8$ treated nano-AIN have a better dispersion than untreated and MPTS treated particles. From DSC results, composites containing particles with polar surface chemistry can result in higher crystallisation temperature, i.e. a stronger nucleating effect, while all samples retain a comparable melting peak.

The dielectric responses of surface treated nanocomposites are very different from the untreated nanocomposites. The imaginary permittivity of MPTS-M samples show relaxation peaks in the low-frequency region $(<1 \mathrm{~Hz})$. The results of $\mathrm{C} 8$ E treated samples shows a flatter upturn of real permittivity and lower imaginary permittivity at low frequencies compared to the untreated nanocomposites. Meanwhile, a significant upturn can be observed in the real and imaginary permittivity of $\mathrm{C} 8$ $\mathrm{M}-10$, which has the same organofunctional groups as $\mathrm{C} 8-\mathrm{E}$ treated nanocomposites. These results indicate that the different hydrolysable groups will also lead to a change in bulk properties, which may be the direct result of the different chemical structure on the near-particle side of surface functionalised nanoparticles. It is consistent with the FTIR results, which show the far-particle side of C8-E and C8-M treated particles to be identical.

The thermal conductivity of nanocomposites increases as a function of the loading ratio. By altering the surface chemistry of nano-AlN, the phonon scattering phenomenon is seemingly reduced. The surface functionalisation can increase compatibility between filler and matrix material, and the different surface treatments seem to have a comparable effect on the thermal conductivity of composite materials. 
In this study, the potential influence of surface chemistry on the near-particle side and a proposed chemical structure of surface functionalised nanoparticle are highlighted. This is very different from the conventional opinion that the distinct layered interphase region exerts the property change. Consequently, future work will require in-depth characterisation of the filler surface chemistry.

\section{REFERENCES}

[1] M. F. Frechette, M. L. Trudeau, H. D. Alamdar, and S. Boily, "Introductory remarks on nanodielectrics," IEEE Trans. on Dielectr. and Electr. Insul., vol. 11, no. 5, pp. 808-818, 2004.

[2] T. Lewis, "Nanometric dielectrics," IEEE Trans. on Dielectr. and Electr. Insul., vol. 1, no. 5, pp. 812-825, 1994.

[3] J. K. Nelson, J. C. Fothergill, L. A. Dissado, and W. Peasgood, "Towards an understanding of nanometric dielectrics," in IEEE Conf. Electr. Insul. Dielectr. Phenomena (CEIDP), 2002, pp. 295-298.

[4] M. Roy, J. Nelson, R. MacCrone, L. S. Schadler, C. Reed, and R. Keefe, "Polymer nanocomposite dielectrics-the role of the interface," IEEE Trans. on Dielectr. and Electr. Insul., vol. 12, no. 4, pp. 629-643, 2005.

[5] S. Raetzke and J. Kindersberger, "The effect of interphase structures in nanodielectrics," IEEJ Trans. Fundam. Mater., vol. 126, no. 11, pp. 1044-1049, 2006.

[6] K. Y. Lau, "Structure and electrical properties of silica-based polyethylene nanocomposites," PhD Thesis, University of Southampton, 2013.

[7] Y. Zhou, J. Hu, X. Chen, F. Yu, and J. He, "Thermoplastic polypropylene/aluminum nitride nanocomposites with enhanced thermal conductivity and low dielectric loss," IEEE Trans. on Dielectr. and Electr. Insul., vol. 23, no. 5, pp. 2768-2776, 2016.

[8] W. Peng, X. Huang, J. Yu, P. Jiang, and W. Liu, "Electrical and thermophysical properties of epoxy/aluminum nitride nanocomposites: Effects of nanoparticle surface modification," Compos. Part A-Appl. Sci. Manuf., vol. 41, no. 9, pp. 1201-1209, 2010.

[9] E. P. Plueddemann, Silane coupling agents. Springer Boston MA, 1991.

[10] Y. Xie, C. A. S. Hill, Z. Xiao, H. Militz, and C. Mai, "Silane coupling agents used for natural fiber/polymer composites: A review," Compos. Part A-Appl. Sci. Manuf., vol. 41, no. 7, pp. 806-819, 2010.

[11] C. Yeung, "Spectroscopic Analysis of Nanodielectric Interfaces," PhD Thesis, University of Southampton, 2013.

[12] I. Hosier, M. Praeger, A. Holt, A. Vaughan, and S. Swingler, "On the effect of functionalizer chain length and water content in polyethylene/silica nanocomposites: Part I-Dielectric properties and breakdown strength," IEEE Trans. on Dielectr. and Electr. Insul., vol. 24, no. 3, pp. 1698-1707, 2017.

[13] S. Zhao, L. Cheng, J. Leng, L. Guo, Y. Gao, Y. Zheng, and D. Cao, "Synergistic enhancement of glass fiber and tetrapod-shaped $\mathrm{ZnO}$ whisker on the mechanical and thermal behavior of isotactic polypropylene," J. Appl. Polym. Sci., vol. 133, no. 46, p. 44217, 2016.

[14] M. Abdelmouleh, S. Boufi, M. N. Belgacem, and A. Dufresne, "Short natural-fibre reinforced polyethylene and natural rubber composites: effect of silane coupling agents and fibres loading," Compos. sci. and technol., vol. 67, no. 7, pp. 1627-1639, 2007.

[15] M. Roy, J. K. Nelson, R. MacCrone, and L. Schadler, "Candidate mechanisms controlling the electrical characteristics of silica/XLPE nanodielectrics," J. Mater. Sci., vol. 42, no. 11, pp. 3789-3799, 2007.

[16] T. Andritsch, A. Vaughan, and G. C. Stevens, "Novel insulation materials for high voltage cable systems," IEEE Electr. Insul. Mag., vol. 33, no. 4, pp. 27-33, 2017.

[17] R. Kochetov, T. Andritsch, U. Lafont, P. Morshuis, S. Picken, and J. Smit, "Thermal behaviour of epoxy resin filled with high thermal conductivity nanopowders," in IEEE Electr. Insul. Conf. (EIC), 2009, pp. 524-528.

[18] X. Wang, T. Andritsch, and G. Chen, "Effect of Surface Functionalization on the Dielectric Properties of Polypropylene Aluminium Nitride Nanocomposites," in IEEE Internat. Conf. on Dielectr. (ICD), 2018.

[19] I. Hosier, M. Praeger, A. Vaughan, and S. Swingler, "The effects of water on the dielectric properties of aluminum based nanocomposites," IEEE Trans. on Nanotech., vol. 16, no. 4, pp. 667-676, 2017.
[20] E. Senis, I. O. Golosnoy, O. Thomsen, J. Barton, and S. Madsen, "Characterization of through-thickness thermal conductivity of wind turbine blade CFRP materials using a steady-state technique," in Internat. Conf. on Compos. Mater., 2017.

[21] C. Bressy, V. G. Ngo, F. Ziarelli, and A. Margaillan, "New insights into the adsorption of 3-(trimethoxysilyl) propylmethacrylate on hydroxylated $\mathrm{ZnO}$ nanopowders," Langmuir, vol. 28, no. 6, pp. 3290$3297,2012$.

[22] F. Kremer and A. Schönhals, Broadband Dielectric Spectroscopy. Springer-Verlag Berlin Heidelberg, 2012.

[23] L. A. Dissado and R. M. Hill, "Anomalous low-frequency dispersion. Near direct current conductivity in disordered low-dimensional materials," J. Chem. Soc., Faraday Trans. 2: Molecular and Chemical Physics, vol. 80, no. 3, pp. 291-319, 1984.

[24] R. Ayoob, "Dielectric properties of hexagonal boron nitride polymer nanocomposites," PhD Thesis, University of Southampton, 2017.

[25] T. S. English, J. C. Duda, J. L. Smoyer, D. A. Jordan, P. M. Norris, and L. V. Zhigilei, "Enhancing and tuning phonon transport at vibrationally mismatched solid-solid interfaces," Phys. Rev. B, vol. 85, no. 3, p. 035438, 2012.

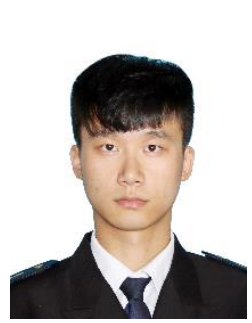

Xinyu Wang was born in Xi'an, China, in 1992. He received the BEng degree from Dalian Maritime University, Dalian, China, in 2015 and the MSc degree from the University of Southampton, UK, in 2016. Currently, he is a PhD student in the University of Southampton. His research interesting is nanodielectrics and surface functionalisation.



Thomas Andritsch (M '11) was born in Innsbruck, Austria, in 1980. He received the Dipl.-Ing. degree in electrical engineering from Graz University of Technology in 2006 and his $\mathrm{PhD}$ in the same field from Delft University of Technology in 2010. Since 2013 he has been a lecturer at the University of Southampton with a research focus on advanced insulation materials for high voltage applications. He has extensive experience with preparation and testing of polymerbased electrical insulation materials, including nanodielectrics, electroactive polymers, and syntactic foams. Andritsch is currently chair of the UK and Ireland Chapter of the IEEE DEIS and was member of IEEE and CIGRE working groups focusing on polymer-based nanodielectrics.

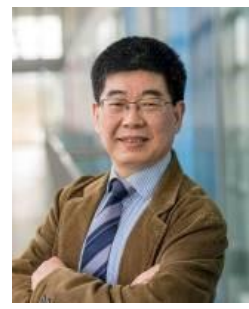

George Chen (SM'11) was born in China in 1961. He received the B.Eng. (1983) and M.Sc. (1986) degrees in electrical engineering from Xi'an Jiaotong University, China. After he obtained the Ph.D. degree (1990) from the University of Strathclyde, UK, on the work of permanent changes in electrical properties of irradiated low-density polyethylene, he joined the University of Southampton as postdoctoral research fellow and became a senior research fellow subsequently. In 1997 he was appointed as a research lecturer and promoted to a Reader in 2002 . He is now the professor of highvoltage engineering at the University of Southampton and a visiting professor of Xi'an Jiaotong University. Over the years, he has developed a wide range of interests in high-voltage engineering and electrical properties of materials and published over 300 papers. He is active in the HVDC systems and involved with technical working groups in both IEEE and CIGRE.

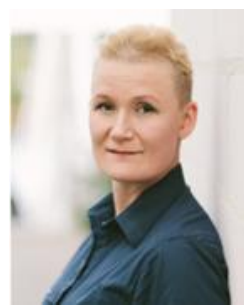

Suvi Virtanen was born in Helsinki, Finland in 1979. She received the M.Sc. degree in organic chemistry from the University of Jyväskylä in 2004 at the international Master's Programme of Nanoscience. From the same university she received the Ph.D. degree in physical chemistry in 2013. Since 2014 she has been Research Fellow at Electronics and Computer Science at the University of Southampton. 